\title{
Designing programs and educational opportunities to aid pediatricians in treating children with mental illness in an outpatient academic pediatric setting
}

\author{
Lidija Petrovic-Dovat ${ }^{1 *}$, Cheston M Berlin ${ }^{2}$, Pevitr S Bansal ${ }^{1}$ and Benjamin N Fogel ${ }^{2 *}$ \\ ${ }^{1}$ Department of Psychiatry, Penn State College of Medicine, Hershey, Pennsylvania, USA \\ ${ }^{2}$ Department of Pediatrics, Penn State College of Medicine, Hershey, Pennsylvania, USA
}

\begin{abstract}
Objective: Pediatricians face many barriers in working with children with behavioral and mental health disorders, such as lack of training, resources, and time. We sought to improve the utility of current educational materials and examined the advantages of developing new educational programs, materials, and interventions to assist pediatricians in the diagnosis and treatment of behavioral and mental health disorders in their patients.
\end{abstract}

Methods: Two "Research Electronic Data Capture" (REDCap) surveys were administered to pediatricians, residents and nurse practitioners at an outpatient clinic to assess current treatment practices of children with mental illness. Survey 1 had a 52\% response rate, while survey 2 had a $61 \%$ response rate.

Results: Overall, results on both surveys demonstrated that pediatricians reported difficulty in monitoring referrals and coordinating services as the most challenging aspect of behavioral health treatment. In addition, they also reported that they were not routinely screening for anxiety disorders. Hence, we implemented an evidenced-based anxiety screening instrument to well-child exams for children aged 11 and older. We also conducted several lectures highlighting depression, anxiety, and suicide prevention.

Conclusions: Pediatricians reported that the anxiety screening helped them identify patients with anxiety disorders. They also reported an interest in learning about other forms of mental health (e.g., depression, anxiety, suicide), which has led to the ongoing development of web-based lectures and online educational resources (e.g., evidence-based scales). Future goals are to continue to aid pediatric providers in treating children with mental illness.

\section{Introduction}

Designing Programs and Educational Opportunities to Aid Pediatricians in Treating Children with Mental Illness in an Outpatient Academic Pediatric Setting.

It is estimated that $11-20 \%$ of children in the United States meet the criteria for a behavioral disorder, and about half of the individuals that will develop a mental illness will experience the initial symptoms by the age of 14 years [1]. In fact, psychiatric illness has long been associated with shorter life expectancies. For example, suicide is the second leading cause of death among individuals 15-34 years-old. Furthermore, shorter life expectancies are also associated with psychiatric disorders such as depression (11 years shorter), bipolar disorder and schizophrenia (20 years shorter) [2], and drug and/or alcohol abuse (24 years shorter). Up to $40 \%$ of children will be diagnosed with at least two mental health disorders, thus substantially increasing the complexity of diagnosis and treatment $[1,3]$. Further, psychiatric illness in children can negatively impact quality of life and, within the physically ill, can further impair functioning and adherence to treatment, thereby worsening comorbid medical conditions $[4,5]$.

Availability of behavior health specialists is limited, and only about $25 \%$ of children with a mental illness are seen by a behavioral health specialist [6]. Consequently, primary care providers are often the first line of care for children with mental and behavioral health issues; however, few primary care providers report confidence in their ability to successfully manage mental illness. Many pediatricians feel ill-equipped within this realm of healthcare (e.g., diagnosing, assessing for patient safety, treatment of mental illness, follow-up care) due to a lack of training, resources, and time [6,7]. Therefore, to identify how pediatricians diagnose and treat mental health illnesses, the challenges they face, and the interventions that are needed to better treat children with behavioral health disorders, we administered surveys to pediatricians. We implemented changes based on the first survey and then, in the second survey, assessed the benefits of the intervention and recommended additional changes.

\section{Methods}

Two investigator-designed online surveys hosted by the secure web application, Research Electronic Data Capture (REDCap) were administered to providers at the primary care and outpatient pediatric

Correspondence to: Lidija Petrovic-Dovat, Department of Psychiatry, Penn State College of Medicine. 22-B Northeast Drive, Rm 24B, Hershey, Pennsylvania, USA, Tel: (717) 531-8338; Fax: (717) 531-6250; Email: lpetrovicdovat@ pennstatehealth.psu.edu

Benjamin N. Fogel, Department of Pediatrics, Penn State College of Medicine, Hershey, Pennsylvania, USA, Email: bfogel@pennstatehealth.psu.edu

Key words: psychiatry, pediatrics, medicine, behavioral health

Received: February 22, 2018; Accepted: March 05, 2018; Published: March 09, 2018 

pediatric setting

clinics. Providers included attending pediatricians, residents, and nurse practitioners. Participation was anonymous and voluntary. Survey 1 was administered in 2014. Of the 29 pediatric providers that received the first survey, 15 responded (response rate $=52 \%$ ). Survey 2 was administered in 2017 to the same group of outpatient pediatric providers and included pediatric residents. Of the 38 pediatricians that received the survey link, 23 responded (response rate $=61 \%$ ).

\section{Results}

\section{Survey 1}

Results from Survey 1 indicated that most pediatricians (61\%) felt that the most challenging aspect within mental healthcare was difficulty coordinating services and monitoring referrals (Table 1). Pediatricians reported attention-deficit hyperactivity disorder (ADHD) as the most common mental health disorder they diagnosed and treated in their practice $(62 \%)$. Most providers (62\%) were not routinely screening for anxiety disorders, yet anxiety was identified as the most common reason for referral to a child psychiatrist (31\%), followed by depression. Hence, we implemented an evidenced-based anxiety screening instrument to well-child exams for children aged 11 and older and in the following survey pediatricians reported that the anxiety screening helped them identify patients with anxiety disorders (Table 2).

Providers were interested in additional educational opportunities that could fit into their busy schedules. This feedback prompted the organization of live lectures for pediatricians. Lecture topics included: a review of evidence-based treatments for anxiety disorders; medication management; treatment of depression in children and adolescents; case study discussions; suicide risk assessments; and confidentiality issues in child and adolescent psychiatry. Each topic was followed by discussion after the lecture. Results from the first survey also led to the creation of the Pediatric Behavioral Health Collaborative Care Program, which was developed by the Department of Child and Adolescent Psychiatry with the implementation of some previously published recommended strategies $[7,8]$. We designed the program and clinic following a previously-described level of integration levels [9].

The consultation clinic utilizes a collaborative model where a child and adolescent psychiatrist provides outpatient consultation to general pediatricians and "curb side consult" for acute issues. Pediatricians are also able to discuss case issues with child and adolescent psychiatrists via phone or in-person. Following feedback from the pediatricians, we expanded these psychiatric services by including follow-up appointments for the patient at the psychiatric clinic. This was done after initial consultation at the pediatric site if the case was too complex, or if pediatricians did not feel comfortable initiation and titrating medications. Patients were seen by a psychiatrist until they were stabilized on medication and were then refereed back to primary care for follow-up after coordinating the plan with the provider.

\section{Survey 2}

In 2017, the results from Survey 2 demonstrated that $72 \%$ of pediatricians referred patients to the consultation clinic we developed. Further, $94 \%$ of pediatricians reported that the consultation clinic was a helpful and positive tool. Pediatricians also indicated that they would prefer easily accessible online video lectures that they could complete during their free time. The topics that pediatricians were most interested in were lectures regarding anxiety disorders (35\%), depression (23\%), and suicide prevention (15\%) and the preferred length of lectures was 30 minutes (Table 3 ).
Table 1. Most challenging aspects for pediatrician.

\begin{tabular}{|l|c|c|}
\hline Aspect & Number & \% of Total $(\boldsymbol{N}=\mathbf{2 4})$ \\
\hline $\begin{array}{l}\text { Lack of exposure to psychiatric patients } \\
\text { during training }\end{array}$ & 1 & 4 \\
\hline $\begin{array}{l}\text { Difficulty in managing patients with } \\
\text { mental health disorders }\end{array}$ & 2 & 8 \\
\hline $\begin{array}{l}\text { The time required to administer screens } \\
\text { and manage cases }\end{array}$ & 4 & 17 \\
\hline $\begin{array}{l}\text { Lack of confidence in successfully } \\
\text { managing behavioral/emotional problems }\end{array}$ & 5 & 21 \\
\hline $\begin{array}{l}\text { Difficulty in coordinating services and } \\
\text { monitoring referrals }\end{array}$ & 10 & 42 \\
\hline Other & 2 & 8 \\
\hline
\end{tabular}

Table 2. Use of anxiety screener.

\begin{tabular}{|l|c|c|}
\hline Aspect & Number & \% of Total $(\boldsymbol{N}=\mathbf{4 1})$ \\
\hline Start treatment & 3 & 7 \\
\hline Diagnose and anxiety disorder & 3 & 8 \\
\hline Refer the patient & 12 & 29 \\
\hline Identify patients with anxiety symptoms & 23 & 56 \\
\hline
\end{tabular}

Table 3. Preferred length of webcast trainings.

\begin{tabular}{|c|c|c|}
\hline Length $^{\mathbf{a}}$ & Number & \% of Total $(\boldsymbol{N}=\mathbf{2 2})$ \\
\hline 15 minutes & 8 & 36 \\
\hline 30 minutes & 13 & 59 \\
\hline 45 minutes & 1 & 5 \\
\hline
\end{tabular}

Note: a Providers were also given an option for a ' 60 minute' webcast training, but this yielded 0 responses.

\section{Discussion}

Pediatricians are often the first line of care in treating children with behavioral and mental health disorders yet feel ill-equipped to treat these complex cases. To assist pediatricians in working within the realm of mental health, our division has developed collaborative care models where mental health services are integrated with primary care, and where mental health specialists provide consultation to medical staff for cases evaluated at the pediatric clinic site. We continue to adjust the program to fit pediatricians' busy outpatient practice. Results from the pilot survey data demonstrate that pediatricians reported a high degree of satisfaction with the collaborative program. In addition, there was an increase in the number of pediatricians who addressed patients' anxiety symptoms during the visit.

Results from this study should be interpreted considering a few limitations. First, our sample size was relatively small for both surveys and was only administered at a single pilot site. In addition, the survey was designed by our investigators and was not validated prior to being administered to the providers. Given these limitations, future studies should sample a larger number of providers at multiple sites to examine if there are increases in the number of diagnoses and referrals after including screening instruments at well-child exams.

Despite these limitations, our results highlight how pediatricians are actively interested in learning more about mental health disorders. Our proposed web-based, open-access educational video series is just one method in disseminating this information in a larger and more efficient manner. Thus, the creation of this web-series is ongoing and will be worth investigation to examine the effects of this online application. This ongoing effort is another way in which we are attempting to close the gap between pediatric and mental healthcare. 
Petrovic-Dovat L (2018) Designing programs and educational opportunities to aid pediatricians in treating children with mental illness in an outpatient academic pediatric setting

\section{References}

1. Merikangas KR, He JP, Burstein M, Swanson SA, Avenevoli S, et al. (2010) Lifetime prevalence of mental disorders in U.S. adolescents: Results from the national comorbidity survey replication-adolescent (NSC-A). J Am Acad Child Adolesc Psychiatry 49: 980-989. [Crossref]

2. Chesney E, Goodwin GM, Fazel S (2014) Risks of all cause and suicide mortality in mental disorders: A meta-review. World Psychiatry 13: 153-160. [Crossref]

3. Costello E J, Mustillo S, Erkanil A, Keeler G, Angold A (2003) Prevalence and development of psychiatric disorders in childhood and adolescence. Arch Gen Psychiatry 60: 837-844. [Croosref]

4. DeMaso DR, Martini DR, Cahen LA (2009)Practice parameter for the psychiatric assessment and management of physically ill children and adolescents. $J$ Am Acad Child Adolesc Psychiatry 48: 213-233. [Crossref]
5. Macartney SE (2011) Child poverty in the United States 2009 and 2010: Selected race groups and Hispanic origin.

6. Weitzman C, Wegner, L (2015) Promoting optimal development: Screening for behavioral and emotional problems. Pediatrics 135: 384-395. [Crossref]

7. Ginsburg, S., Foster, S., Santoro, K., Schoeman, J., \& Chockley, N. (2009). Strategies to support the integration of mental health into pediatric primary care. National Institute for Health Care Management.

8. Richardson LP, Ludman E, McCauley E, Lindenbaum J, Larison C, et al. (2014) Collaborative care for adolescents with depression in primary care: A randomized clinical trial. JAMA 312: 809-816. [Crossref]

9. Heath B, Wise RP, Reynolds KA (2013) A standard framework for levels of integrated healthcare. In. Washington, DC: SAMHSA-HRSA Center for Integrated Health Solutions.

Copyright: (C2018 Petrovic-Dovat L. This is an open-access article distributed under the terms of the Creative Commons Attribution License, which permits unrestricted use, distribution, and reproduction in any medium, provided the original author and source are credited. 